\title{
The dynamically hot stellar halo around NGC 3311: a small cluster-dominated central galaxy $\star, \star \star$
}

\author{
G. Ventimiglia ${ }^{1,2}$, O. Gerhard ${ }^{1}$, M. Arnaboldi ${ }^{2,3}$, and L. Coccato ${ }^{1}$ \\ 1 Max-Plank-Institut für Extraterrestrische Physik, Giessenbachstraße 1, 85741 Garching bei München, Germany \\ e-mail: gventimi@mpe.mpg.de \\ 2 European Southern Observatory, Karl-Schwarzschild-Straße 2, 85748 Garching bei München, Germany \\ 3 INAF, Osservatorio Astronomico di Pino Torinese, 10025 Pino Torinese, Italy
}

Received 27 July 2010 / Accepted 10 September 2010

\section{ABSTRACT}

\begin{abstract}
Context. An important open question is the relation between intracluster light and the halos of central galaxies in galaxy clusters. Aims. Here we report results from an on going project with the aim to characterize the dynamical state in the core of the Hydra I (Abell 1060) cluster around NGC 3311.

Methods. We analyze deep long-slit absorption line spectra reaching out to $\sim 25 \mathrm{kpc}$ in the halo of NGC 3311.

Results. We find a very steep increase in the velocity dispersion profile from a central $\sigma_{0}=150 \mathrm{~km} \mathrm{~s}^{-1}$ to $\sigma_{\text {out }} \simeq 450 \mathrm{~km} \mathrm{~s}{ }^{-1}$ at $R \simeq 12 \mathrm{kpc}$. Farther out, to $\sim 25 \mathrm{kpc}, \sigma$ appears to be constant at this value, which is $\sim 60 \%$ of the velocity dispersion of the Hydra I galaxies. With its dynamically hot halo kinematics, NGC 3311 is unlike other normal early-type galaxies.

Conclusions. These results and the large amount of dark matter inferred from X-rays around NGC 3311 suggest that the stellar halo of this galaxy is dominated by the central intracluster stars of the cluster, and that the transition from predominantly galaxy-bound stars to cluster stars occurs in the radial range 4 to $12 \mathrm{kpc}$ from the center of NGC 3311 . We comment on the wide range of halo kinematics observed in cluster central galaxies, depending on the evolutionary state of their host clusters.
\end{abstract}

Key words. galaxies: clusters: general - galaxies: clusters: individual: Hydra I - galaxies: kinematics and dynamics galaxies: individual: NGC 3311

\section{Introduction}

An important open question is the physical and evolutionary relation between the intracluster light (ICL) and the extended halo of the brightest cluster galaxies (BCGs), whether they are truly independent components or whether the former is a radial extension of the latter. Using a sample of 683 SDSS clusters Zibetti et al. (2005) found a surface brightness excess with respect to an inner $R^{1 / 4}$ profile that characterizes the mean profile of the BCGs, but it is not yet known whether this cD envelope is simply the central part of the cluster's diffuse light component, or whether it is distinct from the ICL and part of the host galaxy (Gonzalez et al. 2005).

In the Southern hemisphere, the cD galaxy NGC 3311 and the giant elliptical NGC 3309 dominate the central region of the Hydra I cluster, an X-ray bright, non-cooling flow, medium compact cluster with a velocity dispersion $\sigma_{\text {HydraI }}=784 \mathrm{~km} \mathrm{~s}^{-1}$ (Misgeld et al. 2008). The X-ray observations show that the hot intracluster medium centered on NGC 3311 has a fairly uniform distribution of temperature and metal abundance from a few kpc out to a radius of $230 \mathrm{kpc}$ (Tamura et al. 2000; Yamasaki et al. 2002; Hayakawa et al. 2004, 2006). Given the overall regular $\mathrm{X}$-ray emission and temperature profile, the Hydra I cluster is considered as a prototype of an evolved and dynamically relaxed

^ Based on observations collected at the European Organisation for Astronomical Research in the Southern Hemisphere, Chile, during the observing program 082.A-0255(A) on 2009 March 25.

$\star \star$ Table 1 is only available in electronic form at http://www . aanda.org cluster; it is therefore a suitable candidate for a dynamical study of a relaxed extended stellar halo around a BCG.

The primary goal of this work is to establish the dynamical state of the stellar halo of NGC 3311. We use long-slit spectra to uncover the kinematics in the halo region of NGC 3311 out to $\sim 25 \mathrm{kpc}$ from its center. In Sect. 2 we present observations with FORS2 at VLT and the GEMINI GMOS archive data, which we reanalyze. We describe the data reduction and the kinematic measurements in Sect. 3. The newly measured halo kinematics and their implications are discussed in Sect. 4, and our conclusions are summarized in Sect. 5.

We adopt a distance to NGC 3311 of 51 Mpc (NED), equivalent to a distance modulus of 33.54 mag. Then $1^{\prime \prime}$ corresponds to $0.247 \mathrm{kpc}$.

\section{Observations and archive data}

VLT FORS2 long slit spectra - The long-slit spectra were obtained during the nights of 2009 March 25-28, with FORS2 on VLT-UT1. The instrumental setup had a long-slit 1."6 wide and 6.8 long, Grism 1400V+18, with instrumental dispersion of $0.64 \AA$ pixel $^{-1}$ and spectral resolution $\sigma=90 \mathrm{~km} \mathrm{~s}^{-1}$, and a spatial resolution along the slit of 0.252 pixel $^{-1}$. The seeing during observations ranged from 0.7 to 1 '.2 2 . The wavelength coverage of the spectra is from $4655 \AA$ to $5965 \AA$, including absorption lines from $\mathrm{H}_{\beta}, \mathrm{MgI}(\lambda \lambda 5167,5173,5184 \AA)$ and Fe I ( $\lambda \lambda 5270,5328 \AA)$. We obtained eight spectra of $1800 \mathrm{~s}$ each, for a total exposure time of $4 \mathrm{~h}$. In the FORS2 observations, the long slit is centered on the dwarf galaxy HCC 26 at $\alpha=10^{\mathrm{h}} 36^{\mathrm{m}} 45.85^{\mathrm{s}}$ and $\delta=-27^{\mathrm{d}} 31^{\mathrm{m}} 24.2^{\mathrm{s}}$ (J2000), with a 


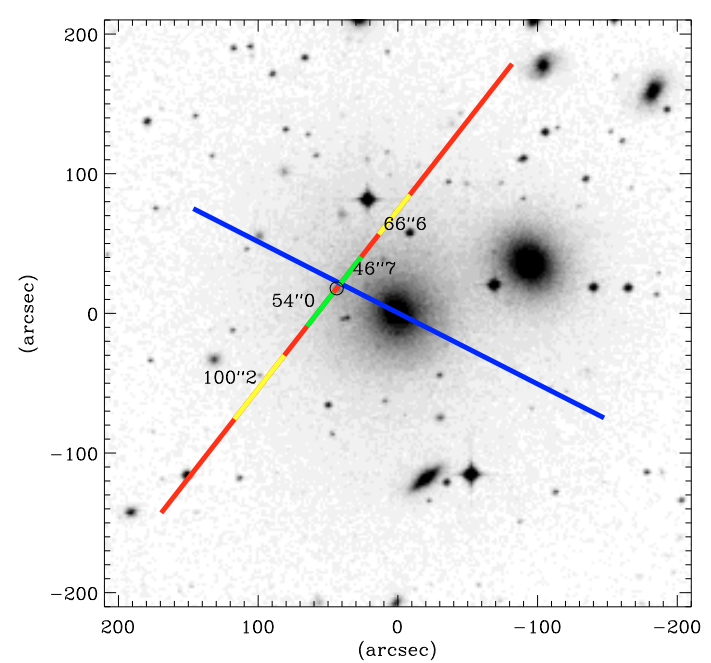

Fig. 1. Optical DSS $7^{\prime} \times 7^{\prime}$ image centered on NGC 3311 in the Hydra I cluster. The relative positions of the GMOS slit $(5 \cdot 5$, blue line) and FORS2 slit (6.8, red line) are illustrated. Green and yellow sections on the FORS2 slit indicate regions where average spectra are extracted. The adjacent numbers specify the radial distances of their lightweighted mean positions from the center of NGC 3311. The center of the FORS2 slit coincides with the position of the dwarf galaxy HCC 26 and is marked by a black circle. North is up and East to the left.

position angle of $\mathrm{PA}=142^{\circ}$; HCC 26 is seen in projection onto the NGC 3311 halo. The position of the FORS2 long slit is shown in Fig. 1. Its center is located at $\mathrm{PA}=64^{\circ}$ with respect to NGC 3311, approximately along the major axis of the galaxy.

Gemini GMOS-South long slit spectra - We use Gemini archive long-slit spectra in the wavelength range from $3675 \AA$ to $6266 \AA$ observed with the $\mathrm{B} 600$ grating, a dispersion of $0.914 \AA$ pixel $^{-1}$, a spectral resolution of $\sigma=135 \mathrm{~km} \mathrm{~s}^{-1}$, and a spatial scale of $0^{\prime} .146$ pixel $^{-1}$; a detailed description of the instrumental setup is presented in Loubser et al. (2008). The seeing was typically in the range from $0{ }^{\prime} 6$ to $1 . \prime 2$. We target the same absorption lines as for the FORS2 spectra, i.e. $\mathrm{H}_{\beta}$, $\operatorname{MgI}(\lambda \lambda 5167,5173,5184 \AA)$ and Fe I $(\lambda \lambda 5270,5328 \AA)$. The 0.5 wide and 5.5 long Gemini slit is centered on NGC 3311 , at $\alpha=10^{\mathrm{h}} 36^{\mathrm{m}} 42.74^{\mathrm{s}}$ and $\delta=-27^{\mathrm{d}} 31^{\mathrm{m}} 41.3^{\mathrm{s}}$ (J2000), along $\mathrm{PA}=63^{\circ}$, the direction of the galaxy major axis. Its position is shown in Fig. 1.

\section{Data reductions}

The data reduction of the FORS2 long slit spectra is carried out in IRAF. After the standard operations of bias subtraction and flat-fielding, the spectra are registered, co-added and wavelength calibrated. The edges of the FORS2 slit reach well into sky regions, which are then used to interpolate the sky emission in the regions covered by the stellar spectra.

In the low surface brightness regions, spectra are summed along the spatial direction in order to produce one-dimensional spectra with an adequate $S / N$ ratio ( $\geq 20$ per $\AA$ ). Four independent one-dimensional spectra are extracted along the slit where the light is dominated by the halo of NGC 3311; of these, two are from regions north and two from regions south of HCC 26, respectively. We extract spectra from slit regions of $\sim 31^{\prime \prime} \times 1^{\prime \prime}$. 6 and $\sim 25^{\prime \prime} \times 11^{\prime \prime} .6$ at distances of about $54^{\prime \prime}$ and $47^{\prime \prime}$ from the center of NGC 3311 , and of $\sim 58^{\prime \prime} \times 11^{\prime \prime} 6$ and $\sim 36^{\prime \prime} \times 11^{\prime \prime} 6$ at central
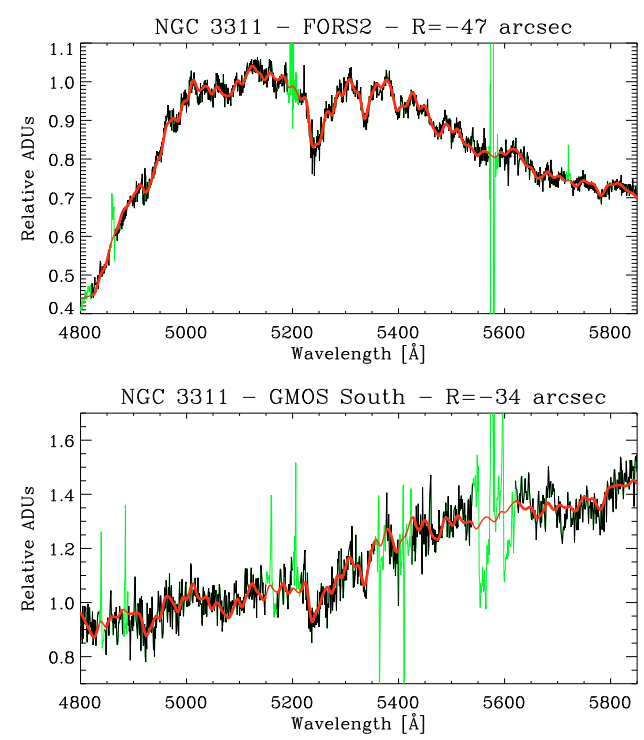

Fig. 2. Kinematic fits with PPXF of the spectra extracted at $-47^{\prime \prime}$ (VLT FORS2) and at -34" (Gemini GMOS). In black we display the galaxy spectra, in green the wavelength range excluded from the fit because of sky residuals, and in red the best-fit-broadened template model. All spectra are normalized to the value of the best-fit model at $5100 \AA$.

distances of about $100^{\prime \prime}$ and $67^{\prime \prime}$. We properly mask the spectra of foreground stars in those areas.

The data reduction for the GMOS long slit spectra is carried out independently here, also in IRAF and with the standard tasks in the Gemini package. The procedure is described in Loubser et al. (2008) for the wavelength calibration and background subtraction; also in this case the edges of the slit are used to interpolate the sky emission in the regions covered by the stellar continuum. Because our goal is to sample the kinematics well into the halo, the one-dimensional spectra for the absorption line measurements are summed along the slit direction so that a minimum $S / N \sim 20$ per $\AA$ is obtained in each radial bin, out to a radial distance of about 40" from the center of NGC 3311.

Stellar kinematics - The stellar kinematics is measured from the extracted 1D spectra in the wavelength range $4800 \AA<\lambda<$ $5800 \AA$, using both the "penalized pixel-fitting" method (PPXF, Cappellari \& Emsellem 2004) and the Fourier correlation quotient (FCQ) method (Bender 1990), in order to account for possible systematic errors and template mismatch.

In the PPXF method, stellar template stars from the MILES library (Sanchez-Blazquez et al. 2007) are combined to fit the one-dimensional extracted spectra; the rotational velocity, the velocity dispersion and Gauss-Hermite moments (e.g. Gerhard 1993) are measured simultaneously. Figure 2 shows two of the extracted spectra and the broadened templates fit by PPXF. In the FCQ method, the rotational velocity and velocity dispersion are derived for each extracted one-dimensional spectrum by assuming that the LOSVD is described by a Gaussian plus third- and fourth- order Gauss-Hermite functions. Before to the fitting procedure the MILES template spectra are smoothed to the GMOS and FORS2 spectral resolution with the measured broadening offsets. While FCQ provides error estimates along with the measured kinematics, errors for the PPXF kinematic parameters are calculated with a series of Monte Carlo simulations adopting the appropriate $S / N$ for each bin.

Because the stellar populations in $\mathrm{cD}$ halos may have different metal abundances and ages from those of the inner regions 


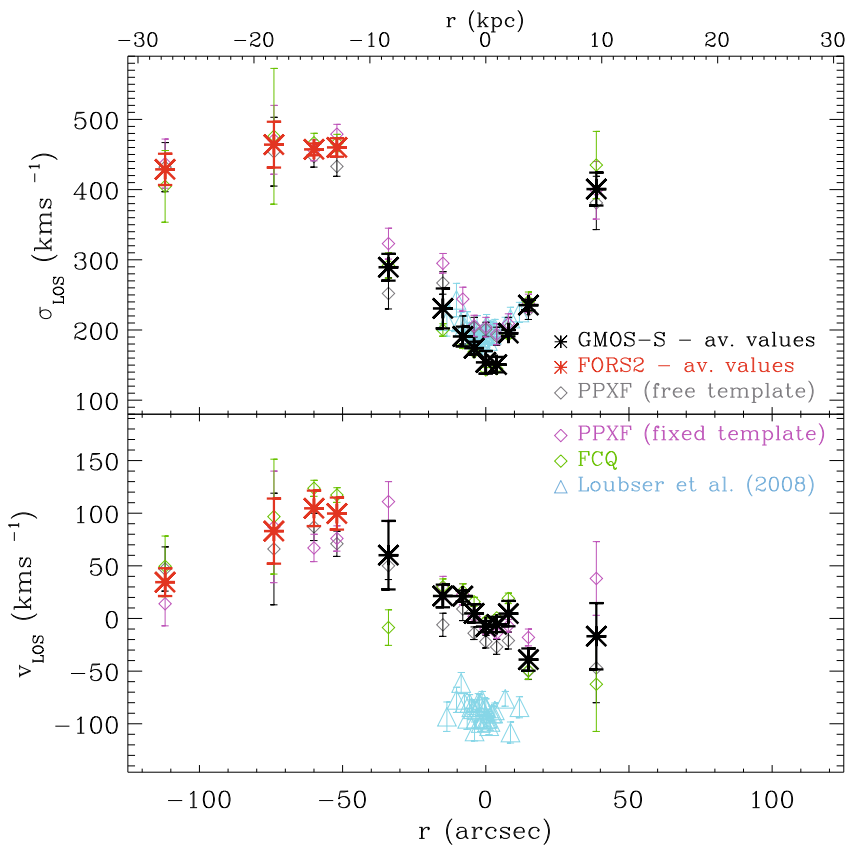

Fig. 3. Major axis line-of-sight velocity and velocity dispersion profiles for NGC $3311\left(\mathrm{PA}=63^{\circ}\right)$. The open light blue triangles are the values published by Loubser et al. (2008), based on Gemini-South GMOS data. The black asterisks are our independent measurements from these GMOS (archival) spectra, and the red asterisks show measurements from the new VLT-FORS2 spectra. These are weighted averages of three independent measurements which are obtained with PPXF and FCQ as described in Sect. 3 and shown separately as the gray, magenta and green diamonds. The FORS2 data points are obtained from averages over $\sim 25^{\prime \prime}$ and $\sim 31^{\prime \prime}$ in the inner regions and over $\sim 36^{\prime \prime}$ and $\sim 58^{\prime \prime}$ in the outer regions of the slit; see Fig. 1. These off-axis measurements are plotted at their light-weighted average radii, corrected for projection onto the major axis of NGC 3311 with an isophotal flattening of 0.89. Positive distances are south-west from the center of NGC 3311 and negative values are north-east, along $\mathrm{PA}=63^{\circ}$.

(Coccato et al. 2010a,b), systematic effects caused by template mismatch must be evaluated and accounted for. We therefore extract kinematic measurements with PPFX and FCQ as follows:

1. fit with PPFX the best stellar template from the MILES library in the central regions with the highest $S / N$, and extract $v$ and $\sigma$ at all radii, using the same stellar template;

2. simultaneously fit the best stellar template, $v$ and $\sigma$ in each radial bin with PPFX;

3. adopt the respective best-fit PPXF stellar template to derive the LOSVD with FCQ for all radial bins;

4. finally, average rotational velocities $v$ and velocity dispersions $\sigma$ are computed as weighted means of the three values extracted in each radial bin as detailed above. Errors for these weighted average values are computed from those of the three measurements, but if the reduced $\chi^{2}=\frac{1}{(n-1)} \sum_{i=1}^{n} \frac{\left(x_{i}-\bar{x}\right)^{2}}{\epsilon_{i}^{2}}$ is greater than one, they are increased by $\sqrt{\chi^{2}}$ in order to take into account systematic differences. I.e., $\epsilon_{\bar{x}}^{2}=\frac{1}{\sum_{i=1}^{n} 1 / \epsilon_{i}^{2}} \times \chi^{2}$ where $\epsilon_{i}, \epsilon_{\bar{x}}$ are the errors on the individual measurements $x_{i}$ and the weighted mean $\bar{x}$, respectively.

Mean velocities and velocity dispersions in all radial bins are listed in Table 1, and the profiles are shown in Fig. 3 together with the previous measurements from Loubser et al. (2008). Table 1, which is available in electronic form, contains the following information: source of data (Col. 1), distance from galaxy center (Col. 2), PA (Col. 3), $v, \sigma$ with errors for each of the procedures 1.-4. described in the text, in Col. (4-5), (6-7), (8-9), and (10-11), respectively. Heliocentric and relativistic corrections have been applied to the mean velocities. The systemic velocity is $3800 \mathrm{~km} \mathrm{~s}^{-1}$ and has been subtracted.

In the central region of NGC 3311, our new velocity dispersion profile marginally agrees with that of Loubser et al. (2008). The new mean line-of-sight velocity measurements agree with the systemic velocity of NGC 3311 obtained by Misgeld et al. (2008), but have a systematic offset from the $v$ data of Loubser et al. (2008), by about $91 \mathrm{~km} \mathrm{~s}^{-1}$. The agreement between the new FORS2 measurements at $-47^{\prime \prime}$ and the revised value at $-34^{\prime \prime}$ from archive GMOS data gives us confidence that the systematic effects from wavelength calibration offsets, template mismatch, etc., are sufficiently small in the new, independent data reductions. However, several tests have convinced us that the data do not allow us to reliably determine full line-ofsight distributions (e.g., $h_{3}, h_{4}$ ), which could be used to test for subcomponents, which one would expect in particular at radii $\sim 30^{\prime \prime}-40^{\prime \prime}$.

\section{The kinematics of the NGC 3311 stellar halo}

The combined new velocity dispersion profile for NGC 3311 reaches to $R_{m j}=39^{\prime \prime} \simeq 10 \mathrm{kpc}$ from the center of NGC 3311 along the galaxy's major axis $\left(\mathrm{PA}=63^{\circ}\right)$, and to an off-axis distance of $R=100^{\prime \prime} \simeq 25 \mathrm{kpc}$ along the FORS2 slit. It shows a very unusual steep rise with increasing radial distance from the galaxy center: from a central value $\sigma_{0}=150 \mathrm{~km} \mathrm{~s}^{-1}$, to $\sigma=231 \mathrm{~km} \mathrm{~s}^{-1}$ at $R=15^{\prime \prime} \simeq 3.7 \mathrm{kpc}$, and then on to a flat $\sigma_{\text {out }} \simeq 450 \mathrm{~km} \mathrm{~s}^{-1}$ outside $R=47^{\prime \prime}=12 \mathrm{kpc}$. The steep outward gradient is supported by two independent data sets and data reductions. The measurements of Loubser et al. (2008) near the galaxy center had already hinted at a positive gradient from $190 \mathrm{~km} \mathrm{~s}^{-1}$ at $R=5^{\prime \prime}$ to $\sim 240 \mathrm{~km} \mathrm{~s}^{-1}$ at a radius of $R=10^{\prime \prime}$, and data shown in Fig. 1 of Hau et al. (2004) reach $\simeq 300 \mathrm{~km} \mathrm{~s}^{-1}$ at $\sim 25^{\prime \prime}$. With the new data we now have very clear evidence of a dynamically hot stellar halo in NGC 3311.

To put the extremely rapid rise of the velocity dispersion profile of NGC 3311 in context, we compare its kinematic properties with those of early-type galaxy (ETG) halos mapped using planetary nebula data (Coccato et al. 2009) and with the halos of the two Coma BCG galaxies NGC 4889, NGC 4874 from deep absorption line spectroscopy (Coccato et al. 2010a). Figure 4 shows the mean $\langle V / \sigma\rangle$, X-ray luminosity, and total absolute $B$-band magnitude for these galaxies versus their outermost halo velocity dispersion. For NGC 3311, we use a bolometric X-ray luminosity within $50^{\prime \prime} \simeq 12 \mathrm{kpc}, L_{\mathrm{X}}=2.75 \times 10^{40} \mathrm{erg} \mathrm{s}^{-1}$ (based on the flux in the $0.5-4.5 \mathrm{keV}$ energy range from Yamasaki et al. (2002) and corrected to bolometric $L_{X}$ according to Table 1 of O'Sullivan et al. 2001), and the total extinction corrected $B$-band magnitude (12.22) from de Vaucouleurs et al. (1991). For the velocity dispersion of NGC 3311, we use the values at the center, at $15^{\prime \prime}(\simeq 3.7 \mathrm{kpc})$ and at $47^{\prime \prime}(\simeq 12 \mathrm{kpc})$. Only the central $\sigma_{0}$ puts NGC 3311 in the middle of the ETG distribution; $\sigma\left(47^{\prime \prime}\right)$ deviates strongly, with a much larger $\sigma$ than expected for the given $L_{X}, B_{T}$.

The natural interpretation for these results is that the outer stellar halo of NGC 3311 is dominated by the central intracluster star component of the Hydra cluster. This is supported by several pieces of evidence: (i) The steep rise of the $\sigma$-profile; more isolated ETGs all have slightly or even steep falling $\sigma$-profiles (Coccato et al. 2009); (ii) The saturation of $\sigma$ at $\simeq 12 \mathrm{kpc}$, outside of which the dynamically hot component dominates 


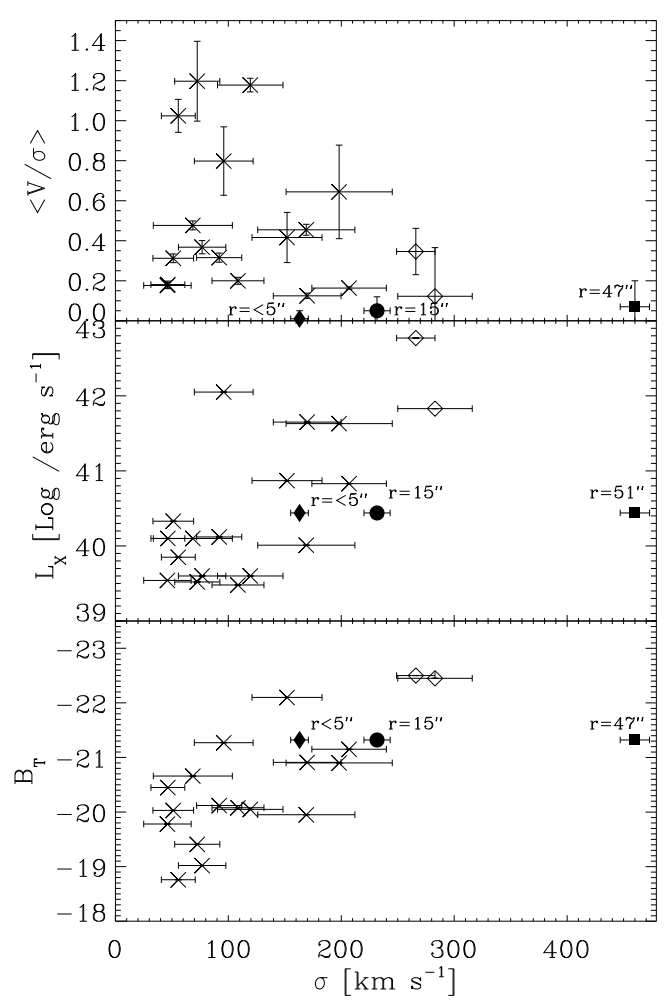

Fig. 4. Properties of the stellar halo of NGC 3311 compared with other early-type galaxy halos: mean $\langle V / \sigma\rangle$ (upper panel), total X-ray luminosity (central panel), and $B$-band total magnitude (lower panel) against stellar velocity dispersion $\sigma$. Solid diamond, circle, and square show the measured $\sigma$ of NGC 3311 at the center, $15^{\prime \prime}(\simeq 3.7 \mathrm{kpc})$, and $47^{\prime \prime}$ ( $\simeq 12 \mathrm{kpc}$ ). Crosses show outermost velocity dispersions from Coccato et al. (2009), and open diamonds for NGC 4889/4874 from Coccato et al. (2010a).

completely. $\sigma\left(47^{\prime \prime}\right)$ is $\sim 60 \%$ of the galaxy velocity dispersion in the cluster core; (iii) The large amount of dark matter inferred from X-ray observations around NGC $3311\left(\sim 10^{12} M_{\odot}\right.$ within $20 \mathrm{kpc}$, Hayakawa et al. (2004)).

In recent cosmological hydrodynamic simulations of cluster formation, Dolag et al. (2010) applied a kinematic decomposition to the stellar particles around $\mathrm{cD}$ galaxies. With a double Maxwellian fit to the velocity histogram of star particles centered on a simulated $\mathrm{cD}$, they were able to separate an inner, colder Maxwellian distribution associated with the central galaxy, and an outer, hotter component of stars that orbit in the cluster potential. For both components they derived radial density profiles and, fitting Sersic profiles, found that the inner stellar component is much steeper than the outer diffuse stellar component. A comparison with these simulations indicates that the steep velocity dispersion gradient in the halo of NGC 3311 traces the transition from central galaxy stars to the diffuse intracluster stellar component. In the NGC 3311 halo, the transition between the two occurs at smaller radii than in other BCGs in nearby clusters, in the range between 4 and $12 \mathrm{kpc}$.

NGC 3311 appears to have a similar halo as the $\mathrm{cD}$ galaxy NGC 6166 in the Abell 2199 cluster (Kelson et al. 2002), whose $\sigma$-profile rises to cluster values at $R \sim 60 \mathrm{kpc}$. But NGC 3311 is even more extreme; it is a fairly small galaxy, based on its central $\sigma_{0}=150 \mathrm{~km} \mathrm{~s}^{-1}$, and it is already dominated by the surrounding cluster component at $R \sim 12 \mathrm{kpc}$. Presumably, this is because the core of the "relaxed" Hydra cluster has had time to collapse onto the galaxy. For comparison, the two BCG galaxies in the Coma cluster core, which have a nearly constant $\sigma$-profile (Coccato et al. 2010a), may be in the middle of an ongoing merger (Gerhard et al. 2007), so that their previous subcluster halos would have been stripped and a new cluster halo could have been built only after the merger was completed; and in the outer halo of the more isolated M 87, the velocity dispersion appears to drop (Doherty et al. 2009) towards the edge.

\section{Conclusions}

Based on two independent long-slit data sets and reductions, we find a steep gradient in the velocity dispersion profile of the central galaxy NGC 3311 in the Hydra I cluster, from $\sigma_{0} \simeq$ $150 \mathrm{~km} \mathrm{~s}^{-1}$ to $\sigma_{\text {out }} \simeq 450 \mathrm{~km} \mathrm{~s}^{-1}$ outside $12 \mathrm{kpc}(60 \%$ of the velocity dispersion of the galaxies in the surrounding cluster).

The new data provide evidence that NGC 3311 is a fairly small galaxy dominated by a large envelope of intracluster stars already beyond $R \sim 12 \mathrm{kpc}$, whose orbits are dominated by the cluster dark matter potential. Comparison with other BCG galaxies shows a wide range of dynamical behavior in their halos.

Acknowledgements. The authors wish to thank the ESO VLT staff for their support during the observations and the referee for a constructive report. This research has made use of the Gemini archive data, the NASA/IPAC Extragalactic Database (NED), which is operated by the Jet Propulsion Laboratory, California Institute of Technology, under contract with the National Aeronautics and Space Administration.

\section{References}

Bender, R. 1990, A\&A, 229, 441

Cappellari, M., \& Emsellem, E. 2004, PASP, 116, 138

Coccato, L., Arnaboldi, M., Gerhard, O., et al. 2010a, A\&A, 519, A95

Coccato, L., Gerhard, O., \& Arnaboldi, M. 2010b, MNRAS, L97

Coccato, L., Gerhard, O., Arnaboldi, M., et al. 2009, MNRAS, 394, 1249

de Vaucouleurs, G., de Vaucouleurs, A., Corwin, Jr., H. G., et al. (Eds.) 1991,

Third Reference Catalogue of Bright Galaxies, Vol.1-3 (New York: Springer)

Doherty, M., Arnaboldi, M., Das, P., et al. 2009, A\&A, 502, 771

Dolag, K., Murante, G., \& Borgani, S. 2010, MNRAS, 405, 1544

Gerhard, O., Arnaboldi, M., Freeman, K. C., et al. 2007, A\&A, 468, 815

Gerhard, O. E. 1993, MNRAS, 265, 213

Gonzalez, A. H., Zabludoff, A. I., \& Zaritsky, D. 2005, ApJ, 618, 195

Hau, G. K. T., Hilker, M., Bridges, T., et al. 2004, in Outskirts of Galaxy

Clusters: Intense Life in the Suburbs, ed. A. Diaferio, IAU Coll., 195, 491

Hayakawa, A., Furusho, T., Yamasaki, N. Y., Ishida, M., \& Ohashi, T. 2004, PASJ, 56, 743

Hayakawa, A., Hoshino, A., Ishida, M., et al. 2006, PASJ, 58, 695

Kelson, D. D., Zabludoff, A. I., Williams, K. A., et al. 2002, ApJ, 576, 720

Loubser, S. I., Sansom, A. E., Sánchez-Blázquez, P., Soechting, I. K., \& Bromage, G. E. 2008, MNRAS, 391, 1009

Misgeld, I., Mieske, S., \& Hilker, M. 2008, A\&A, 486, 697

O'Sullivan, E., Forbes, D. A., \& Ponman, T. J. 2001, MNRAS, 328, 461

Sanchez-Blazquez, P., Peletier, R. F., Jimenez-Vicente, J., et al. 2007, VizieR Online Data Catalog, 837, 10703

Tamura, T., Makishima, K., Fukazawa, Y., Ikebe, Y., \& Xu, H. 2000, ApJ, 535, 602

Yamasaki, N. Y., Ohashi, T., \& Furusho, T. 2002, ApJ, 578, 833

Zibetti, S., White, S. D. M., Schneider, D. P., \& Brinkmann, J. 2005, MNRAS, 358,949

Page 5 is available in the electronic edition of the journal at http: //www . aanda . org 
Table 1. Measured mean velocities and velocity dispersions for NGC 3311.

\begin{tabular}{|c|c|c|c|c|c|c|c|c|c|c|}
\hline Instr. & $\begin{array}{c}R \\
(\operatorname{arcsec}) \\
(2) \\
\end{array}$ & $\begin{array}{l}\text { PA } \\
\text { (3) } \\
\end{array}$ & $\begin{array}{c}V_{\mathrm{ppxf1}} \\
\left(\mathrm{km} \mathrm{s}^{-1}\right) \\
(4)\end{array}$ & $\begin{array}{c}\sigma_{\mathrm{ppxf1}} \\
\left(\mathrm{km} \mathrm{s}^{-1}\right) \\
(5)\end{array}$ & $\begin{array}{c}V_{\mathrm{ppxf2} 2} \\
\left(\mathrm{~km} \mathrm{~s}^{-1}\right) \\
(6)\end{array}$ & $\begin{array}{c}\sigma_{\mathrm{ppxf2}} \\
\left(\mathrm{km} \mathrm{s}^{-1}\right) \\
(7)\end{array}$ & $\begin{array}{c}V_{\mathrm{FCQ}} \\
\left(\mathrm{km} \mathrm{s}^{-1}\right) \\
(8)\end{array}$ & $\begin{array}{c}\sigma_{\mathrm{FCQ}} \\
\left(\mathrm{km} \mathrm{s}^{-1}\right) \\
(9)\end{array}$ & $\begin{array}{c}\langle V\rangle \\
\left(\mathrm{km} \mathrm{s}^{-1}\right) \\
(10)\end{array}$ & $\begin{array}{c}\langle\sigma\rangle \\
\left(\mathrm{km} \mathrm{s}^{-1}\right) \\
(11)\end{array}$ \\
\hline GMOS-S & 38.6 & $63^{\circ}$ & $38 \pm 33$ & $399 \pm 38$ & $-47 \pm 33$ & $381 \pm 38$ & $-62 \pm 45$ & $435 \pm 48$ & $-17 \pm 31$ & $401 \pm 23$ \\
\hline GMOS-S & 14.89 & $63^{\circ}$ & $-18 \pm 8$ & $238 \pm 13$ & $-50 \pm 8$ & $228 \pm 13$ & $-49 \pm 8$ & $241 \pm 14$ & $-39 \pm 11$ & $235 \pm 8$ \\
\hline GMOS-S & 7.88 & $63^{\circ}$ & $-7 \pm 8$ & $209 \pm 15$ & $-21 \pm 8$ & $203 \pm 15$ & $19 \pm 5$ & $193 \pm 5$ & $5 \pm 12$ & $195 \pm 5$ \\
\hline GMOS-S & 3.8 & $63^{\circ}$ & $-14 \pm 7$ & $194 \pm 13$ & $-27 \pm 7$ & $191 \pm 13$ & $1 \pm 3$ & $145 \pm 3$ & $-5 \pm 7$ & $151 \pm 11$ \\
\hline GMOS-S & 0 & $63^{\circ}$ & $-10 \pm 6$ & $204 \pm 10$ & $-22 \pm 6$ & $201 \pm 10$ & $-3 \pm 3$ & $143 \pm 3$ & $-8 \pm 5$ & $154 \pm 16$ \\
\hline GMOS-S & -4.09 & $63^{\circ}$ & $2 \pm 6$ & $206 \pm 14$ & $-14 \pm 6$ & $204 \pm 14$ & $16 \pm 4$ & $169 \pm 4$ & $5 \pm 9$ & $174 \pm 9$ \\
\hline GMOS-S & -8.05 & $63^{\circ}$ & $23 \pm 11$ & $244 \pm 20$ & $9 \pm 11$ & $200 \pm 20$ & $26 \pm 7$ & $182 \pm 8$ & $21 \pm 5$ & $191 \pm 14$ \\
\hline GMOS-S & -15 & $63^{\circ}$ & $29 \pm 11$ & $295 \pm 16$ & $-6 \pm 11$ & $267 \pm 16$ & $30 \pm 7$ & $200 \pm 9$ & $22 \pm 11$ & $231 \pm 28$ \\
\hline GMOS-S & -34 & $63^{\circ}$ & $111 \pm 13$ & $323 \pm 22$ & $50 \pm 13$ & $252 \pm 22$ & $-8 \pm 17$ & $292 \pm 18$ & $60 \pm 33$ & $289 \pm 19$ \\
\hline FORS2 & -47 & $45^{\circ}$ & $76 \pm 12$ & $479 \pm 14$ & $71 \pm 12$ & $433 \pm 14$ & $117 \pm 7$ & $466 \pm 12$ & $100 \pm 15$ & $460 \pm 13$ \\
\hline FORS2 & -54 & $83^{\circ}$ & $67 \pm 13$ & $456 \pm 15$ & $87 \pm 13$ & $447 \pm 15$ & $124 \pm 8$ & $467 \pm 14$ & $105 \pm 17$ & $457 \pm 8$ \\
\hline FORS2 & -67 & $6^{\circ}$ & $87 \pm 53$ & $471 \pm 49$ & $66 \pm 53$ & $454 \pm 49$ & $97 \pm 55$ & $476 \pm 97$ & $83 \pm 31$ & $464 \pm 33$ \\
\hline FORS2 & -100 & $114^{\circ}$ & $14 \pm 21$ & $437 \pm 35$ & $47 \pm 21$ & $432 \pm 35$ & $50 \pm 29$ & $405 \pm 51$ & $35 \pm 13$ & $429 \pm 22$ \\
\hline
\end{tabular}

Notes. For details see text. The galaxy's systemic velocity $V_{\text {sys }}=3800 \mathrm{~km} \mathrm{~s}^{-1}$ has been obtained by a linear fit to the velocities in the central $20^{\prime \prime}$ and has then been subtracted from the measurements. This value includes heliocentric and relativistic corrections.

Column 1: instrument; Col. 2: radial distance from center of NGC 3311; Col. 3: position angle of data with respect to NGC 3311's center; Col. 4: velocity measured with PPXF (using template determined at $R=00^{\prime \prime}$ ), relative to the galaxy systemic velocity; Col. 5: velocity dispersion measured with PPXF (using template determined at $R=00^{\prime \prime}$ ); Col. 6: velocity measured with PPXF (free template), relative to the galaxy systemic velocity; Col. 7: velocity dispersion measured with PPXF (free template); Col. 8: velocity measured with FCQ, relative to the galaxy systemic velocity; Col. 9: velocity dispersion measured with FCQ; Col. 10: weighted average velocity; Col. 11: weighted average velocity dispersion. 